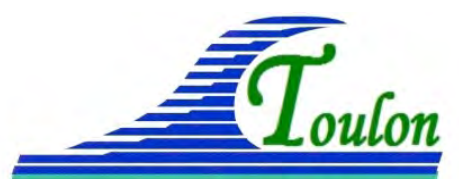

XIV èmes Journées Nationales Génie Côtier - Génie Civil Toulon, 29 juin au $1^{\text {er }}$ juillet 2016

DOI:10.5150/jngcgc.2016.007 C Editions Paralia CFL

disponible en ligne - http://www.paralia.fr - available online

\title{
Formulation semi analytique de la propagation non linéaire de la houle
}

\author{
Mohamed LOUKILI ${ }^{1}$, Soumia MORDANE ${ }^{1}$, Mohamed CHAGDALI ${ }^{1}$
}

\author{
1. Université Hassan II Casablanca - Faculté des Sciences Ben M’Sik - LPPPC, \\ Casablanca, Maroc. \\ mordanesoumia@yahoo.fr
}

\section{Résumé :}

Dans le cadre de la théorie potentielle, le problème de la déformation de la houle se ramène à la recherche de $\varphi$ (potentiel des vitesses) et $\eta$ (forme de la surface libre) qui vérifient l'équation de Laplace dans le domaine fluide et les conditions aux limites sur la surface libre et le fond.

Les méthodes de perturbation constituent une façon d’obtenir des équations équivalentes au problème de départ. Dans ces méthodes, on utilise un développement en série de perturbation par rapport à un paramètre $\varepsilon$ supposé petit. Il présente l'ordre de grandeur de l'amplitude sur la longueur d'onde (cambrure). En cherchant la solution sous la forme d'un développement asymptotique de $(\varepsilon)$, on obtient, à partir du problème de départ, une série de problèmes aux différents ordres en $(\varepsilon)$ qu’on résout d'une manière séquentielle.

Dans ce travail, on montre qu'on peut construire, une formulation récurrente à l'ordre $n$, sous la forme la forme générale :

$L_{n}=L\left(\varphi_{n}(x, y, z)\right)=S\left(\varphi_{n-1}, \varphi_{n-2}, \ldots\right)$

$L_{n}$ est un opérateur aux dérivées partielles du second ordre qui correspond à l'ordre $n$. $S$ est un second membre qui ne contient que les informations aux ordres inférieurs à $n$ en $(\varepsilon)$.

Une fois la solution à l'ordre $n=1$ est connue, les solutions aux différents ordres s'obtiennent en inversant une seule fois l'opérateur $L$. Le gain en temps de calcul est important puisqu'on inverse une seule fois l’opérateur $L$. La résolution numérique se fait par une méthode des différences finis sur un maillage orthogonal et évolutif.

Mots-clés : Propagation de la houle, Modèle non linéaire.

\section{Introduction}

Nous présentons une méthode de perturbation couplée avec la méthode des différences finies pour la résolution du problème de la propagation de la houle dans un canal. Dans ce travail, on propose une méthode semi analytique ou plutôt semi numérique pour la résolution de la propagation non linéaire de la houle. Le principe de la démarche est d'appliquer la méthode de perturbation sur le problème non-linéaire du départ ce qui nous permet d'avoir des problèmes linéaires vérifiés pour les différents ordres 
(NAYFEY, 1973 ; HINSH, 1991). Ces problèmes non linéaires présentent aussi une grande difficulté qui concerne l'écriture des conditions aux limites sur la surface libre dans un domaine évolutif. Les problèmes étant instationnaires, ces transformations numériques seront donc évolutives. La résolution numérique des problèmes linéaires transformés est effectuée par la méthode des différences finies. On présente des solutions pour les ordres supérieurs (ordre 1, ordre 2, ...). Une comparaison avec la solution exacte de stokes est présentée (SUSBIELLES et al., 1981).

\section{Formulation théorique}

Le problème de la houle potentielle se ramène à déterminer le potentiel $\varphi$ et la surface libre $\eta$ tels que:

$$
\begin{aligned}
& \Delta \varphi=0 \quad \text { dans le domaine fluide } \\
& \frac{\partial \varphi}{\partial y}=0 \quad \text { sur le fond } y=-h \\
& \frac{\partial \varphi}{\partial t}+\frac{1}{2}\|\vec{\nabla} \varphi\|^{2}+g \eta=0 \quad \text { sur la surface libre } y=\eta(x, t) \\
& \frac{\partial \varphi}{\partial y}=\frac{\partial \eta}{\partial t}+\frac{\partial \eta}{\partial x} \frac{\partial \varphi}{\partial x} \quad \text { sur la surface libre } y=\eta(x, t)
\end{aligned}
$$

Avec: $(x, y)$ les coordonnées cartésiennes, $h$ la profondeur d'eau; $g$ la pesanteur et $\vec{\nabla}$ l'opérateur gradient.

A ces équations s'ajoutent les conditions initiales, les conditions sur le potentiel en amont et en aval (ORLANSKY, 1976).

\section{Formulation analytique}

Les méthodes de perturbation constituent une façon d’obtenir des équations équivalentes au problème de départ. Dans ces méthodes, on utilise un développement en série de perturbation par rapport à un paramètre $\varepsilon$ supposé petit, donc l'ordre de grandeur est proportionnel à la cambrure de la surface libre (NAYFEY, 1973 ; HINSH, 1991).

$\varepsilon=O\left(\frac{a}{\lambda}\right)$

Où $\lambda$ est la longueur d'onde et $a$ l'amplitude de la houle.

Plus précisément le potentiel de vitesse $\varphi$ et l'élévation de la surface libre $\eta$ sont cherchés sous la forme :

$\varphi=\varphi_{0}+\varepsilon \varphi_{1}+\varepsilon^{2} \varphi_{2}+\ldots$

$\eta=\eta_{0}+\varepsilon \eta_{1}+\varepsilon^{2} \eta_{2}+\ldots$

En injectant (6)-(7) dans les équations (1)-(4), on définit les inconnues $\left(\varphi_{n}, \eta_{n}\right)$ qui seront solutions de $n$ problèmes linéaires bien posés. Ces problèmes linéaires ainsi obtenus, sont écrits dans des domaines physiques à frontière courbe (surface libre). On note le domaine $\mathrm{D}_{\mathrm{i}}$, le domaine physique qui correspond à l'ordre $\mathrm{i}$. 


\section{XIV ${ }^{\text {èmes }}$ Journées Nationales Génie Côtier - Génie Civil \\ Toulon, 29 juin au $1^{\text {er }}$ juillet 2016}

3.1 Problèmes à l'ordre $1(\mathrm{P} 1)$
$\Delta \varphi_{1}=0$
Dans $\mathrm{D}_{1}$
$\partial \varphi_{1} / \partial \mathrm{y}=0$
Sur le fond;
$\partial \varphi_{1} / \partial \mathrm{t}+\mathrm{g} \eta_{1}=0$
Sur la surface libre $y=\eta_{1}(x, t)$
$\partial \eta_{1} / \partial \mathrm{t}-\partial \varphi_{1} / \partial \mathrm{y}=0$
Sur la surface libre $y=\eta_{1}(x, t)$

On remarque que le problème à l'ordre 1 correspond exactement au problème de la houle linéaire. C'est à dire un problème qui peut être déduit directement du problème de départ en négligeant les termes non-linéaires dans les conditions aux limites sur la surface libre. On obtient alors un problème linéaire écrit directement sur la surface libre sans faire de linéarisation de la géométrie,

\subsection{Problème à l'ordre 2 (P2)}

$\Delta \varphi_{2}=0$

Dans $\mathrm{D}_{2}$;

$\partial \varphi_{2} / \partial \mathrm{y}=0$

Sur le fond

$\partial \varphi_{2} / \partial \mathrm{t}+\mathrm{g} \eta_{2}=\mathrm{SM}_{2}{ }^{1}$

Sur la surface libre $y=\eta_{2}(x, t)$

$\partial \eta_{2} / \partial \mathrm{t}-\partial \varphi_{2} / \partial \mathrm{y}=\mathrm{SM}_{2}{ }^{2}$

Sur la surface libre $y=\eta_{2}(x, t)$

Avec :

$S M_{2}^{1}=-0.5\left(\left(\frac{\partial \varphi_{1}}{\partial x}\right)^{2}+\left(\frac{\partial \varphi_{1}}{\partial y}\right)^{2}\right)$

$S M_{2}^{2}=-\frac{\partial \varphi_{1}}{\partial x} \frac{\partial \eta_{1}}{\partial x}$

La formulation des problèmes aux différents ordres peut être généralisée sous la forme suivante :

3.3 Problème à l'ordre p (Pn)

$\Delta \varphi_{\mathrm{p}}=0$

$\partial \varphi_{\mathrm{p}} / \partial \mathrm{y}=0$

$\partial \varphi_{\mathrm{p}} / \partial \mathrm{t}+\mathrm{g} \eta_{\mathrm{p}}=\mathrm{SM}_{\mathrm{p}}{ }^{1}$

$\partial \eta_{\mathrm{p}} / \partial \mathrm{t}-\partial \varphi_{\mathrm{p}} / \partial \mathrm{y}=\mathrm{SM}_{\mathrm{p}}{ }^{2}$
Dans $\mathrm{D}_{\mathrm{p}}$

Sur le fond;

Sur la surface libre $y=\eta_{p}(x, t)$

Sur la surface libre $y=\eta_{p}(x, t)$

Pour $\mathrm{p}>1$, on distingue 2 cas. Cas 1 avec $\mathrm{p}$ impair :

$$
\begin{aligned}
& S M_{p}^{1}=-0.5 \sum_{k=1}^{k=p-2}\left(\frac{\partial \varphi_{k}}{\partial x} \frac{\partial \varphi_{p-k}}{\partial x}+\frac{\partial \varphi_{k}}{\partial y} \frac{\partial \varphi_{p-k}}{\partial y}\right) \\
& S M_{p}^{2}=-\sum_{k=1}^{k=1}\left(\frac{\partial \varphi_{k}}{\partial x} \frac{\partial \varphi_{p-k}}{\partial x}\right)
\end{aligned}
$$


Cas 2 avec p pair :

$$
\begin{aligned}
& S M_{p}^{1}=-0.5 \sum_{k=1}^{k=p-2}\left(2\left(\frac{\partial \varphi_{k}}{\partial x} \frac{\partial \varphi_{p-k}}{\partial x}+\frac{\partial \varphi_{k}}{\partial y} \frac{\partial \varphi_{p-k}}{\partial y}\right)\right)-\left(\frac{\partial \varphi_{p / 2}}{\partial x}\right)^{2}-\left(\frac{\partial \varphi_{p / 2}}{\partial y}\right)^{2} \\
& S M_{p}^{2}=-\sum_{k=1}^{k=1}\left(\frac{\partial \varphi_{k}}{\partial x} \frac{\partial \varphi_{p-k}}{\partial x}\right)
\end{aligned}
$$

Ces problèmes linéaires possèdent le même opérateur linéaire, seuls les seconds membres et la géométrie du domaine d'étude changent ce qui constitue un avantage en vue de la résolution numérique. Mais il présente les difficultés de la résolution dans des domaines à frontières courbes évolutives.

Afin de surmonter cette dernière difficulté, on va exprimer les différents problèmes obtenus dans un système de coordonnées curvilignes orthogonales et évolutives (CHAGDALI, 1990).

\section{Modélisation numérique}

Afin de faciliter le traitement des conditions aux limites sur ces frontières, on utilisera un système de coordonnées curvilignes orthogonales. Ce système de coordonnée sera construit numériquement. Comme le problème considéré est instationnaire, le système des coordonnées curvilignes orthogonales utilisé sera évolutif. On résout à chaque pas de temps successivement les $\mathrm{n}$ problèmes linéaires en générant le maillage associé au système de coordonnées pour chaque problème et à chaque pas de temps.

La construction d'un système de coordonnées curvilignes, pour définir le maillage, pour des géométries à frontières courbes s’obtient à l'aide des méthodes de transformation du domaine physique en domaine rectangulaire dans le cas bidimensionnel.

Nous supposons maintenant que l'application $\boldsymbol{T}$ qui transforme la région physique (D) en une région rectangulaire $\left(D^{t}\right)$ (voir figure 1 ) telle que :

$T: \mathrm{M}(\mathrm{x}, \mathrm{y}) \in \mathrm{D} \rightarrow \mathrm{N}(\xi, \zeta) \in \mathrm{D}^{\mathrm{t}}$ est une bijection.

On suppose qu'à l'instant $t_{n}=n$.dt on est dans la configuration illustrée sur la figure 1 .

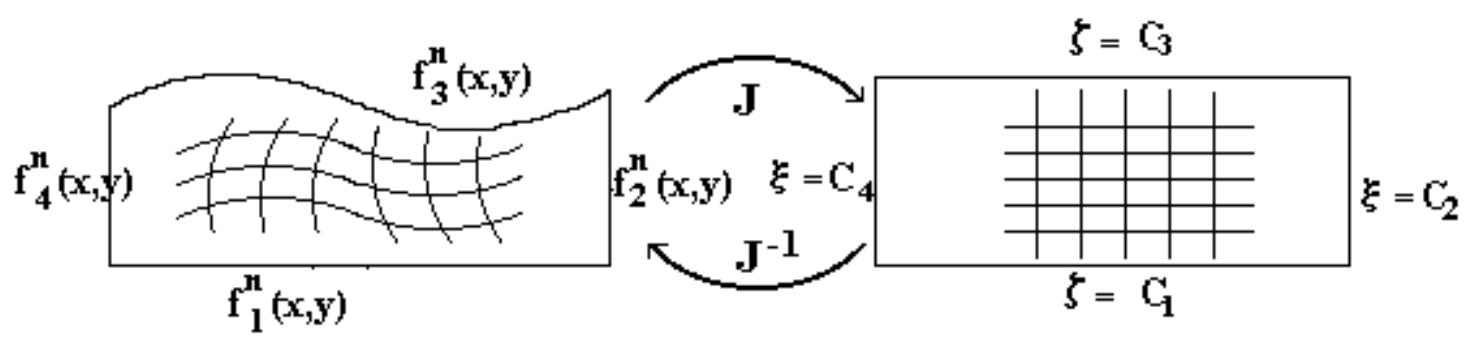

Figure 1. Génération du maillage. 


\section{XIV ${ }^{\text {èmes }}$ Journées Nationales Génie Côtier - Génie Civil \\ Toulon, 29 juin au $1^{\text {er }}$ juillet 2016}

Les opérateurs différentiels qui interviennent dans les équations du mouvement peuvent être exprimés dans le système de coordonnées curvilignes orthogonales $(\xi, \zeta)$ de la manière suivante :

$\Delta_{x, y}(F)=J^{2} \Delta_{\xi \zeta}(F)$
$\vec{\nabla}(\mathrm{F})=\sqrt{\mathrm{J}} \cdot \overrightarrow{\nabla^{*}}\left(\mathrm{~F}^{*}\right)$

où $\mathrm{J}$ est le module de transformation.

La surface libre est évolutive et par conséquent le système de coordonnées curvilignes orthogonales $(\xi, \zeta)$ dépend explicitement du temps. Cette variation temporelle est introduite directement dans les équations du mouvement ce qui fait apparaître des termes supplémentaires dans les parties instationnaires, on peut écrire que :

$\left.\left.\frac{\partial F}{\partial}\right)_{x_{i}}=\frac{\partial F}{\partial t}\right)_{\xi_{i}}+\vec{\nabla}^{*}(F) \cdot U_{d}^{*}$

Avec :

$\vec{\nabla}=\left(\frac{\partial}{\partial x}, \frac{\partial}{\partial y}\right) ; U_{d}^{*}=\left(\frac{\partial \xi}{\partial t}, \frac{\partial \zeta}{\partial t}\right)$

La méthode de résolution numérique des équations du mouvement et des équations de génération du maillage est la méthode des différences finies. Pour le traitement des équations elliptiques on utilise le schéma des directions alternées implicite (A.D.I.). Les conditions aux limites de la surface libre sont traitées par un schéma de CranckNicholson (CHAGDALI, 1990 ; LOC \&DAUBE, 1977).

\section{Résultats}

On se place dans un canal à houle de profondeur constante $\mathrm{h}=2.5 \mathrm{~m}$ et de longueur égale à $21 \mathrm{~m}$. Conformément au domaine de validité de la théorie de Stokes au troisième et cinquième ordre on a fixé la limite supérieure de variation de l'amplitude de $\mathrm{a}: \mathrm{a}<0.4 \mathrm{~m}$.

Les solutions coïncident avec les solutions analytiques jusqu'à l'ordre 3. On remarque que la progression de la houle est périodique dans le temps et qu'il y a variation de la forme dans l'espace. Ces variations se caractérisent d'une part sur la hauteur par rapport au niveau moyen de l'eau au repos et d'autre part par une dissymétrie amont-aval au niveau de la forme de chaque crête. Pour montrer l'efficacité de notre approche, on présente des résultats pour les ordres supérieurs pour différents paramètres de la houle incidente.

La figure 2, présente une comparaison de la solution numérique à l'ordre 5 pour une amplitude incidente a=0,1 m avec la solution de Stokes à l'ordre 5 . On remarque une bonne concordance dans l'ensemble avec une légère dissymétrie amont-aval au niveau de la forme de chaque crête. 


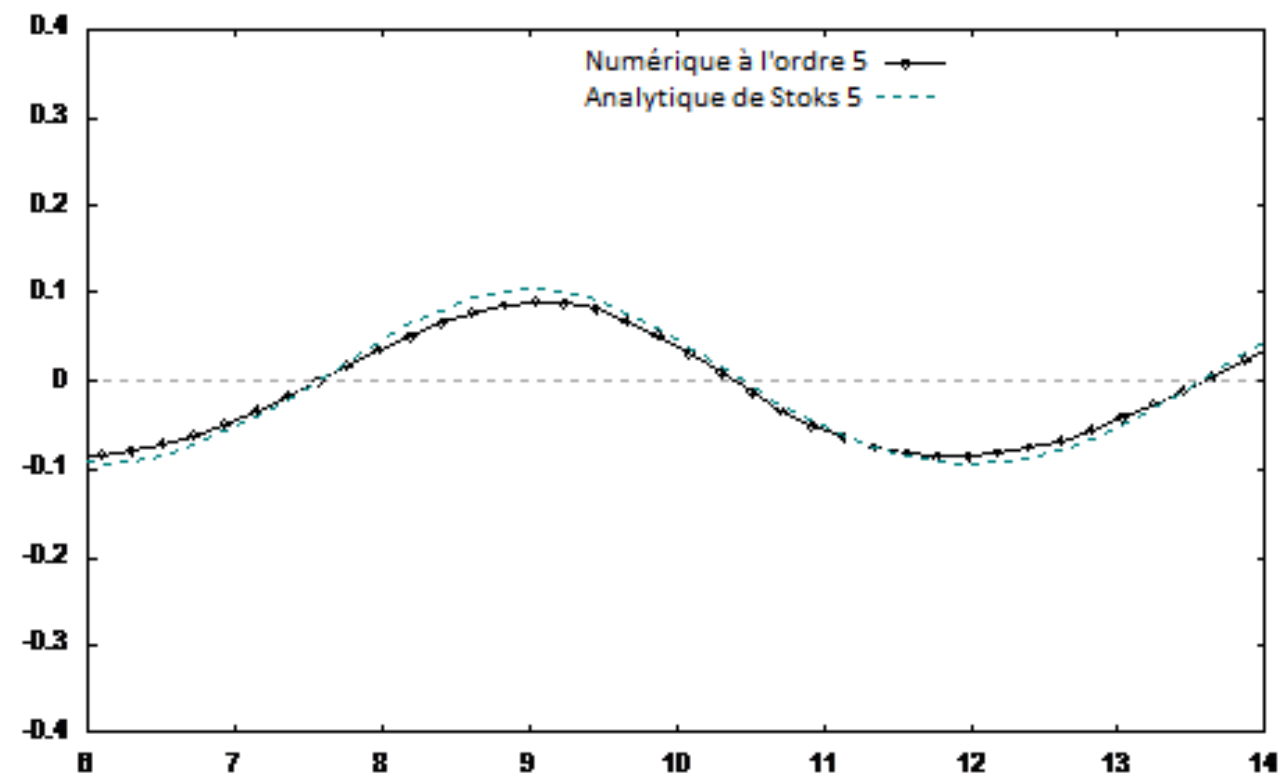

Figure 2. Elévation de la surface libre en fonction de la position le long du canal pour $a=0,1$ m et à l'instant $t=T / 2$.

On présente sur la figure 3 la forme de la surface libre pour des ordres 5, 6 et 7 obtenus numériquement en comparaison avec la solution analytique de Stokes à l'ordre 5.

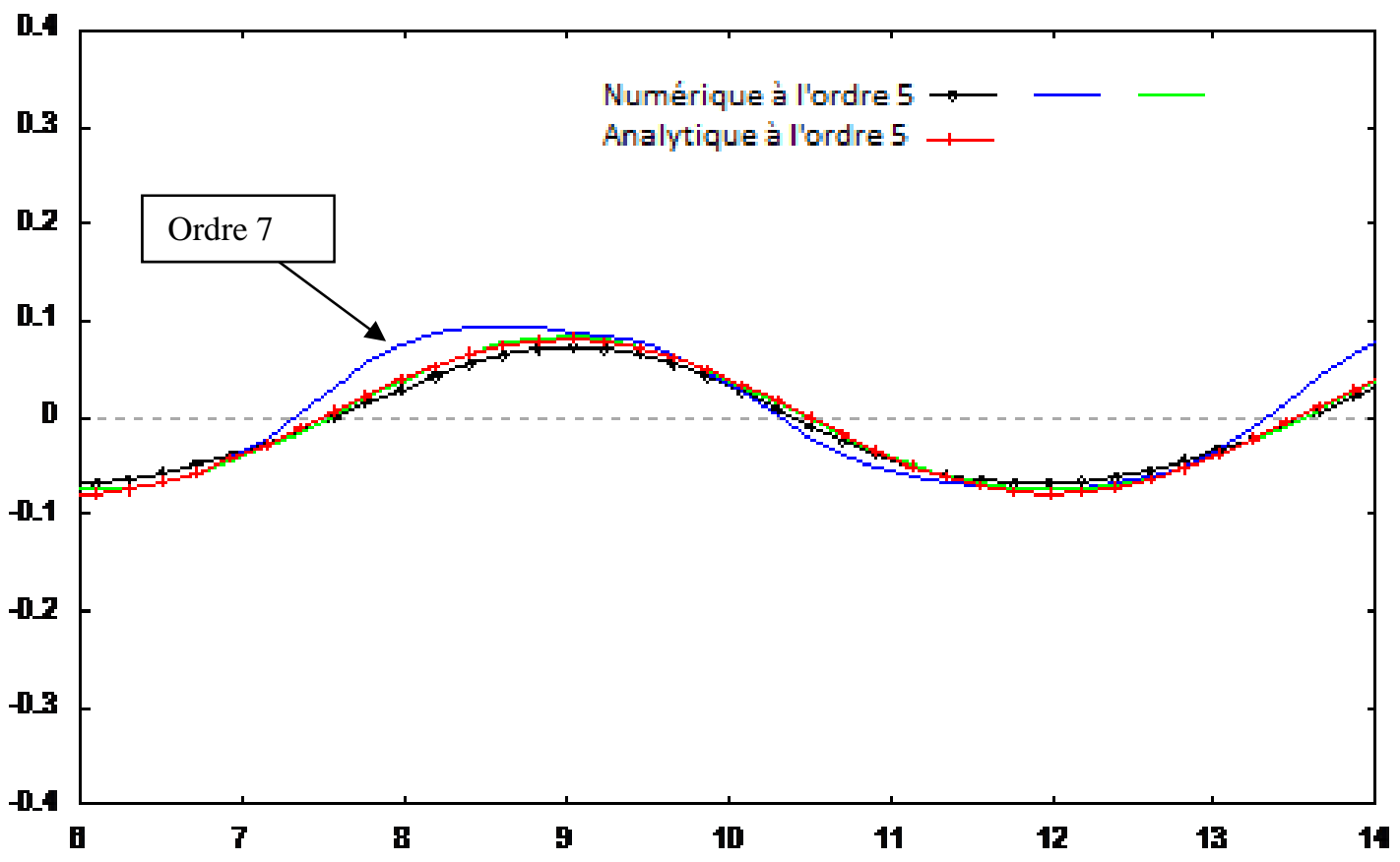

Figure 3. Elévation de la surface libre en fonction de la position le long du canal pour $a=0.08 \mathrm{~m}$. 


\section{XIV èmes Journées Nationales Génie Côtier - Génie Civil \\ Toulon, 29 juin au $1^{\text {er }}$ juillet 2016}

On constate que pour les faibles amplitudes il y a concordance des solutions jusqu'à l'ordre 6. La solution à l'ordre 7 se détache et on a une forte dissymétrie amont-aval au niveau de chaque crête. Pour l'ordre 7, les difficultés sont purement numériques. D’une part, la génération numérique du maillage et son suivi font intervenir le champ de déplacement des nœuds du maillage sur la surface libre et une fois que les déformations augmentent, des erreurs de calcul s'accumulent. D’autre part, les dérivées d'ordres supérieurs qui apparaissent dans les seconds membres, sont difficiles à approcher numériquement.

\section{Conclusion}

Dans ce travail, on a appliqué la méthode semi analytique couplée avec la transformation numérique et orthogonale au problème de la houle non-linéaire et instationnaire. Les courbes solutions ainsi obtenues ont été comparées aux solutions analytiques de Stokes. La comparaison avec une solution exacte a permis de constater que la démarche entreprise est correcte et elle fera l'objet des améliorations en particulier au niveau de la génération du maillage évolutif.

\section{Références bibliographiques}

CHAGDALI M. (1990). Simulation numérique d'écoulement à surface libre d'un fluide visqueux incompressible. Thèse d'Etat de l'Université Hassan II Mohammedia, Maroc.

HINSH E.J. (1991). Perturbation methods. Cambridge Texts in Applied Mathematics, Cambridge University Press, Cambridge.

LOC T.P., DAUBE O. (1977). Une méthode $O\left(h^{2}\right)$ et $O\left(h^{4}\right)$ combinée pour la résolution numérique des équations de Navier-Stokes. Comptes Rendus de l'Académie des Sciences, Série A - Sciences Mathématiques, Vol. 284(19), pp 1241-1243.

NAYFEY A. (1973). Perturbation methods. Eds John Wiley and Sons, New York.

ORLANSKY I. (1976). A simple boundary condition for unbonded hyperbolic flows. J. of Computational Physics, Vol. 21(3), pp 251-269.http://dx.doi.org/10.1016/0021-9991(76)90023-1 SUSBIELLES G., BRATU C., CAVANIE A. (1981). Vagues et ouvrages pétroliers en mer. Publications de l'institut français du pétrole, Editions TECHNIP, Paris, 501 p. 
Thème 1 - Hydrodynamique côtière 